\title{
Prevalência e fatores associados a potenciais interações medicamentosas entre idosos em um estudo de base populacional
}

\author{
Prevalence and factors associated with potential drug interactions among elderly in a population-based study
Prevalencia y factores asociados con potenciales interacciones de drogas entre los ancianos en un estudio de base poblacional

Daniel Riani Gotardelo. Instituto Metropolitano de Ensino Superior (IMES). Ipatinga, MG, Brasil. danielriani@uol.com.br (Autor correspondente) Lorena Silva Fonseca. Instituto Metropolitano de Ensino Superior (IMES). Ipatinga, MG, Brasil. Iorenasf_14@yahoo.com.br Eugênio Rodrigues Masson. Instituto Metropolitano de Ensino Superior (IMES). Ipatinga, MG, Brasil. eugeniomasson@hotmail.com Lauro Nogueira Lopes. Instituto Metropolitano de Ensino Superior (IMES). Ipatinga, MG, Brasil. lauromg@yahoo.com.br Vinícius Nogueira Toledo. Instituto Metropolitano de Ensino Superior (IMES). Ipatinga, MG, Brasil. viniciusnt@hotmail.com Marina Abreu Faioli. Instituto Metropolitano de Ensino Superior (IMES). Ipatinga, MG, Brasil. marinafaioli@gmail.com Aline Martins de Melo Meira. Instituto Metropolitano de Ensino Superior (IMES). Ipatinga, MG, Brasil. aline_m_meira@hotmail.com Cássia Kelly Martins Costa. Instituto Metropolitano de Ensino Superior (IMES). Ipatinga, MG, Brasil. ckmc33@yahoo.com.br Raíssa Braga Linhares Andrade. Instituto Metropolitano de Ensino Superior (IMES). Ipatinga, MG, Brasil. raissabandrade@gmail.com

\section{Resumo}

Objetivo: Determinar a prevalência de potenciais interações medicamentosas e os fatores a elas associados entre idosos cadastrados nas equipes da Estratégia Saúde da Família (ESF) do município de Timóteo, MG. Métodos: Estudo transversal, utilizando amostragem aleatória estratificada. Foram realizadas 273 entrevistas domiciliares, com indivíduos de 60 anos ou mais de idade, por meio de formulário que continha perguntas de identificação, sociodemográficas e questões relacionadas às condições de saúde do idoso e ao consumo de medicamentos. As interações medicamentosas foram identificadas e classificadas de acordo com 0 software Micromedex ${ }^{\circledR}$. Resultados: A prevalência de potenciais interações medicamentosas foi de $55,6 \%$, perfazendo um total de 466 ocorrências, das quais 5,6\% eram leves, 81,6\% eram moderadas e 12,8\% eram de maior gravidade. As classes terapêuticas mais frequentemente envolvidas foram anti-inflamatórios e, principalmente, fármacos utilizados em doenças cardiovasculares. A ausência de internação hospitalar nos últimos 4 meses associou-se significativamente a uma menor chance de interações graves, e a maior parte dos pacientes que não tiveram nenhum tipo de interação moderada utilizava apenas medicamentos prescritos por médicos. Conclusões: A prevalência de interações medicamentosas encontrada foi semelhante à descrita na literatura, demonstrando alta frequência entre idosos. A ausência de internação hospitalar prévia e a prescrição de medicamentos por profissionais médicos associaram-se a uma menor frequência desse fenômeno. A prescrição de múltiplos medicamentos simultaneamente aos idosos pode comprometer a segurança e a saúde dessa população, requerendo, por parte dos cuidadores, observação atenta quanto à ocorrência de interações medicamentosas.

\section{Abstract}

Objectives: The aim of this study was to determine the prevalence of potential drug interactions and the factors associated with them among elderly patients covered by the Family Health Strategy in the municipality of Timóteo, state of Minas Gerais, Brazil. Methods: Cross-sectional study, using stratified random sampling. A total of 273 household interviews were conducted in subjects aged 60 years or older, after obtaining informed consent, using a questionnaire containing questions related to identification, demography, health conditions and medication use. Drug interactions were identified and classified according to the software Micromedex ${ }^{\circledR}$. Results: The overall prevalence of potential drug interactions was $55.6 \%$, a total of 466 cases, of which $5.6 \%$ were mild, $81.6 \%$ moderate and $12.8 \%$ of greater severity. Therapeutic classes most frequently involved were anti-inflammatory drugs and especially drugs used in cardiovascular disease. The absence of hospitalization in the last four months was significantly associated with a lower chance of serious drug interactions and most patients who did not have any moderate drug interactions used only drugs prescribed by physicians. Conclusions: The prevalence of potential drug interactions was similar to that described in the literature, demonstrating the high frequency of this phenomenon among the elderly. The absence of prior hospitalization and drug prescription by physicians were associated with a lower frequency of interactions. The prescription of multiple drugs simultaneously to elderly patients can compromise the safety and health of this population, requiring, by caregivers, observation for the occurrence of potential drug interactions.

Como citar: Gotardelo DR, Fonseca LR, Masson ER, Lopes LN, Toledo VN, Faioli MA et al.

Prevalência e fatores associados a potenciais interações medicamentosas entre idosos em um

estudo de base populacional. Rev Bras Med Fam Comunidade. 2014;9(31):111-8. Disponível em:

http://dx.doi.org/10.5712/rbmfc9(31)833

\section{Palavras-chave:}

Interações de Medicamentos Idoso

Saúde do Idoso Estratégia Saúde da Família Atenção Primária à Saúde

\section{Keywords:}

Drug Interactions Aged Health of the Elderly Family Health Strategy Primary Health Care

Fonte de financiamento: Faculdade de Medicina do Vale do Aço (IMES/Univaço).

Parecer CEP: UnilesteMG 46.267.11.

Conflito de interesses: declaram não haver.

Recebido em: 08/10/2013. Aprovado em: 23/04/2014. 


\section{Resumen}

Objetivo: El objetivo de este estudio fue determinar la prevalencia de posibles interacciones medicamentosas y los factores asociados con ellas en ancianos vinculados a la Estrategia de Salud Familiar en el municipio de Timóteo, MG. Métodos: Estudio transversal, con una muestra aleatoria estratificada. Se realizaron 273 entrevistas domiciliarias en sujetos mayores de 60 años, después del consentimiento de los sujetos de investigación, mediante un cuestionario que contenía preguntas de identificación, demográficos y aspectos relacionados con las condiciones de salud de los ancianos y el consumo de medicamentos. Se identificaron y se clasificaron las interacciones de acuerdo con el software Micromedex ${ }^{\circledR}$. Resultados: La prevalencia global de interacciones potenciales con medicamentos fue del 55,6\%, un total de 466 ocurrencias, de los cuales 5,6\% fueron leves, el 81,6\% moderadas y el 12,8 \% de mayor gravedad. Clases terapéuticas más frecuentemente implicados fueron los fármacos anti-inflamatorios y especialmente fármacos utilizados en la enfermedad cardiovascular. La ausencia de hospitalización en los últimos cuatro meses se asoció significativamente con una menor probabilidad de interacciones graves y la mayoría de los pacientes que no tuvieran ninguna interacción moderada, sólo los medicamentos prescritos por los médicos. Conclusiones: La prevalencia de las interacciones potenciales con el medicamento fue similar a la descrita en la literatura, lo que demuestra la alta frecuencia de este fenómeno entre los ancianos. La ausencia de hospitalización previa y los medicamentos prescritos por los médicos se asociaron con una menor frecuencia de estas interacciones. La prescripción de varios medicamentos al mismo tiempo puede comprometer la seguridad y la salud de esta población, lo que requiere, por los cuidadores, la observación de la aparición de interacciones con medicamentos.
Palabras clave: Interacciones de Drogas Anciano Salud del Anciano Estrategia de Salud Familiar Atención Primaria de Salud

\section{Introdução}

O crescimento da população idosa é uma tendência mundial. Entre 1970 e 2025, espera-se que o aumento do número de idosos seja de 223\%, totalizando 1,2 bilhão de pessoas com mais de 60 anos de idade. Até 2050 haverá dois bilhóes, sendo $80 \%$ nos países em desenvolvimento. No Brasil, entre 1980 e 2000, a populaçáo com 60 anos foi a que mais cresceu com um aumento de 7,3 milhôes, totalizando mais de 14,5 milhôes em 2000. Projeçôes da Organização Mundial da Saúde (OMS) para o ano de 2025 demonstram que o Brasil deverá possuir a $6^{a}$ maior população idosa do mundo e a primeira na América Latina, com cerca de 32 milhōes (15\%) de pessoas com idade acima de 60 anos, o que demandará melhorias no modelo de atenção à saúde prestado no país, sobretudo no tocante às deficiências da assistência farmacêutica prestada a essa população. ${ }^{1-3}$

Sendo marcado por uma elevaçáo da frequência de doenças crônicas náo-transmissíveis, o processo de envelhecimento é acompanhado por uma maior demanda pelos serviços de saúde e por medicamentos, o que predispóe grandemente a população geriátrica aos riscos da polifarmácia, entendida como o consumo simultâneo de múltiplos medicamentos. ${ }^{4}$ Em prescriçóes médicas de idosos, é comum encontrar dosagens inadequadas, interaçóes medicamentosas, associações e redundância - uso de fármacos pertencentes a uma mesma classe terapêutica - além de medicamentos sem valor terapêutico. Isso se deve ao fato de medicamentos poderem ser prescritos para os idosos sem haver clara correspondência entre a doença e a ação farmacológica, sendo muitas vezes empregados de forma equivocada, como consequência de uma cultura de medicalização, impulsionada pelo valor simbólico dado aos medicamentos. ${ }^{5}$ Além disso, os idosos apresentam limitaçóes fisiológicas que podem interferir diretamente nos processos farmacocinéticos (absorção, distribuição, metabolização e eliminação dos medicamentos), podendo os medicamentos, mesmo em doses terapêuticas habituais, produzir efeitos tóxicos e reaçôes medicamentosas adversas. ${ }^{6}$

O uso de vários medicamentos como estratégia terapêutica e o crescente número destes agentes no mercado são fatores que podem contribuir para ampliar os efeitos benéficos da terapia, mas que também possibilitam o aparecimento de efeitos indesejados, tais como aqueles decorrentes de interaçóes medicamentosas. ${ }^{7}$

Interaçôes medicamentosas são situaçôes em que os efeitos de um fármaco modificam-se, ou quando ocorre o surgimento de um novo efeito em decorrência da presença de outro fármaco, algum alimento, bebida ou agente químico ambiental. ${ }^{8}$ Devido a essas potenciais alteraçóes nos efeitos farmacológicos, muitos autores consideram as interaçóes uma forma de reação adversa medicamentosa que, em um conceito mais amplo, pode ser entendida como qualquer malefício causado pela administração de um fármaco. Essas reações são consideradas um problema de saúde pública, sendo responsáveis por 5\% das admissóes hospitalares e estando associadas a um custo médio aproximado de US\$ 16.000 por internação em países desenvolvidos. ${ }^{9-11}$

A ocorrência de interação medicamentosa está fortemente associada ao número de medicamentos utilizados. A polifarmácia é o mais importante fator de risco para a ocorrência de interaçôes medicamentosas: a prevalência desse evento varia de 3 a $5 \%$ na população geral quando se consomem 2 ou 3 medicamentos, chegando a $20 \%$ quando o número de medicamentos consumidos é superior a $10 .{ }^{12}$ 
As interaçôes medicamentosas são mais frequentes em pessoas com idade avançada, ${ }^{13}$ do sexo feminino, ${ }^{7,14}$ em situação conjugal estável (casado), ${ }^{14}$ morando com outra pessoa ${ }^{14}$ e com renda menor ou igual a 3 salários mínimos. ${ }^{14,15} \mathrm{~A}$ ocorrência desse evento é maior ainda entre portadores de doenças crônicas ${ }^{7,13}$ e entre aqueles que fazem uso de automedicaçãa ${ }^{14}-$ situação definida como consumo de um medicamento independentemente da prescrição profissional. ${ }^{16}$ As interaçóes são de gravidade menor ou moderada na maioria das vezes, ${ }^{7,14} \mathrm{e}$ as classes farmacológicas envolvidas mais frequentemente são os fármacos que atuam nos sistemas cardiovascular, gastrointestinal, nervoso e musculoesquelético. ${ }^{14}$

Quanto à gravidade, as interaçóes medicamentosas podem ser classificadas em potencialmente leves, moderadas ou graves. As interaçôes moderadas e graves podem requerer intervenção médica específica para reduzir, respectivamente, a exacerbação de condiçôes clínicas previamente existentes e a possibilidade de efeitos adversos graves. Nem todas as interaçôes medicamentosas são clinicamente relevantes e, mesmo na presença de associaçóes com riscos moderados a graves, antes da substituição ou suspensão das drogas envolvidas, admitem-se a monitorização clínico-laboratorial e a verificação da relação risco-benefício quanto ao seu uso.

Por serem grandes consumidores de medicamentos, os idosos são mais vulneráveis à ocorrência de potenciais interaçóes medicamentosas. Nesse contexto, ressalta-se a importância de determinar a prevalência de potenciais interaçóes medicamentosas e os fatores associados a essa ocorrência (perfil sociodemográfico, indicadores das condiçôes de saúde e do uso de serviços de saúde) nessa população, além de descrever e discutir as interações mais frequentemente encontradas, inclusive em relação ao potencial de gravidade.

\section{Métodos}

Estudo transversal, com amostra aleatória estratificada, representativa dos idosos cadastrados em 15 (quinze) equipes da Estratégia Saúde da Família (ESF) do município de Timóteo, MG. O cálculo de tamanho de amostra estimou uma prevalência de $26,5 \%{ }^{7}$ de potenciais interaçóes medicamentosas, com um nível de confiança de $95 \%$, poder de $80 \%$ e acréscimo de $10 \%$ para eventuais perdas e recusas, sendo proporcional ao número de idosos cadastrados em cada uma das equipes (estrato) do município, de acordo com o último censo realizado pelos agentes comunitários de saúde. A randomização foi realizada utilizando recursos do software Microsoft Excel', 2010. Foram realizadas 273 entrevistas domiciliares com indivíduos de mais de 60 anos de idade, por meio de formulário, após o consentimento dos sujeitos de pesquisa, entre os meses de abril e junho de 2012.

O formulário de pesquisa era constituído de três blocos de perguntas. O bloco A continha perguntas de identificação, sociodemográficas e questôes relacionadas ao consumo de medicamentos que definiram a inclusão ou exclusão do entrevistado nos próximos blocos do estudo. O bloco $\mathrm{B}$ continha perguntas relacionadas às condiçóes de saúde do idoso. O bloco $\mathrm{C}$ contemplava questóes relacionadas ao uso de medicamentos (identificação das substâncias, automedicação, uso inadequado de medicamentos conforme a posologia recomendada).

De acordo com o software Micromedex, ${ }^{17}$ as interaçôes foram classificadas em potencialmente graves (que podem oferecer risco de morte e/ou requerer intervenção médica urgente para minimizar efeitos adversos graves), moderadas (que podem resultar em exacerbação das condiçóes clínicas do paciente e/ou requerer troca de terapia) e leves (interaçóes com efeitos clínicos limitados, podendo sua manifestaçáo incluir aumento da frequência ou severidade dos efeitos adversos, mas que nâo requerem alteraçóes importantes na terapia). ${ }^{18}$

Para a análise de dados, foram construídas tabelas e quadros de distribuição de frequências. Teste t de Student para comparação de médias e teste qui-quadrado de Pearson para comparação de proporçôes foram usados para testar hipóteses de associação entre as covariáveis e o evento na análise univariada. As variáveis associadas ao evento na análise univariada foram submetidas ao modelo multivariado (regressão logística binária). Foram consideradas significativa e independentemente associadas ao evento aquelas variáveis que, no modelo multivariado final, apresentaram valores de $\mathrm{p}<0,05$, isto é, considerou-se um nível de significância de 5\%. O protocolo de pesquisa foi aprovado pelo Comitê de Ética em Pesquisa do Centro Universitário do Leste de Minas Gerais (UnilesteMG), sob o número 46.267.11. 


\section{Resultados}

Foram entrevistados 273 pacientes. Os Quadros 1 e 2 mostram a distribuição de frequência de indicadores de saúde, demográficos e socioeconômicos da populaçáo estudada.

Quadro 1. Distribuição de frequência de indicadores demográficos e socioeconômicos de uma amostra representativa dos idosos cadastrados na ESF do município de Timóteo, MG.

\begin{tabular}{|lll|}
\hline Gênero & Feminino & $58,6 \%$ \\
Faixa etária & $60-69$ anos & $48,7 \%$ \\
Estado civil & Casado & $60,3 \%$ \\
Escolaridade & $1-4$ anos & $55,1 \%$ \\
Renda familiar mensal & $1-2$ salários mínimos & $50,7 \%$ \\
Renda pessoal mensal & $1-2$ salários mínimos & $42,3 \%$ \\
\hline
\end{tabular}

Quadro 2. Distribuição de frequência de indicadores de saúde de uma amostra representativa dos idosos cadastrados na ESF do município de Timóteo, MG.

\begin{tabular}{|lll|}
\hline Número de medicamentos consumidos & $2-4$ & $42,3 \%$ \\
Estado de saúde & Razoável & $42,8 \%$ \\
Consultas médicas & 3 ou mais & $37,2 \%$ \\
Internação hospitalar & Nenhuma & $92,4 \%$ \\
Convênio de saúde & Não possui & $54,9 \%$ \\
Gasto com medicamentos & Sim & $72,8 \%$ \\
\hline
\end{tabular}

Em relação às morbidades encontradas, 79,9\% eram hipertensos, 27,6\% tinham diabetes, 15,4\% doença cardíaca, 13,8\% doença mental, 10,7\% doença reumática, 6,5\% doença pulmonar e 3\% câncer.

A prevalência global de potenciais interaçóes medicamentosas foi de 55,6\%, perfazendo um total de 466 ocorrências encontradas, sendo que 5,6\% eram leves, $81,6 \%$ eram moderadas e $12,8 \%$ eram de maior gravidade.

Potenciais interaçóes medicamentosas leves (PIML) ocorreram em 8,43\% dos entrevistados. Os medicamentos mais frequentes nesse tipo de interação foram ANLODIPINO + IBUPROFENO ( $\mathrm{n}=5$, correspondendo a 21,73\% dos casos de interaçóes leves e a 1,83\% de todos os entrevistados) e ANLODIPINO + DICLOFENACO ( $\mathrm{n}=3)$. Ambas as interaçôes exibiram potencial de redução/antagonismo do efeito anti-hipertensivo. METFORMINA + NIFEDIPINO ( $\mathrm{n}=3)$ poderia ocasionar um aumento da absorção da metformina por mecanismo desconhecido.

Potenciais interações medicamentosas moderadas (PIMM) foram as mais frequentes, ocorrendo em 53,48\% dos entrevistados. Os medicamentos mais frequentemente envolvidos em PIMM foram ÁCIDO ACETILSALICÍLICO + HIDROCLOROTIAZIDA, que ocorreram em 39 entrevistados (26,71\% dos casos de interaçôes moderadas e 14,28\% de todos os entrevistados). Essa interação poderia causar redução da eficácia diurética e anti-hipertensiva por redução das prostaglandinas renais. ENALAPRIL + HIDROCLOROTIAZIDA $(\mathrm{n}=20)$ e CAPTOPRIL + HIDROCLOROTIAZIDA $(n=17)$ foram potenciais interações encontradas com frequência, ambas com possibilidade de gerar hipotensão postural por vasodilatação e depleção do volume intravascular.

Potenciais interaçóes medicamentosas graves (PIMG) ocorreram em 13,98\% de todos os entrevistados. Os medicamentos mais frequentemente envolvidos nas interaçóes graves e as respectivas frequências com que ocorreram foram: ANLODIPINO + SINVASTATINA ( $\mathrm{n}=11$, correspondendo a $26,71 \%$ dos pacientes com PIMG e a $4,02 \%$ de todos os entrevistados), com potencial de causar aumento do risco de efeitos adversos da sinvastatina; DIGOXINA + HIDROCLOROTIAZIDA $(\mathrm{n}=4)$ com aumento do risco de intoxicação digitálica por hipopotassemia e; ENALAPRIL + LOSARTANA ( $\mathrm{n}=3$ ) gerando aumento do risco de hiperpotassemia, hipotensão e disfunção renal. Consta no Quadro 3 um resumo da descrição das potenciais interaçóes medicamentosas encontradas no estudo. 
Quadro 3. Potenciais interações medicamentosas (com o número de pacientes acometidos entre parênteses) encontradas em uma amostra representativa dos idosos cadastrados na ESF do município de Timóteo, MG.

\begin{tabular}{|ccc|}
\hline Potenciais interações medicamentosas leves & Potenciais interações medicamentosas moderadas & Potenciais interações medicamentosas graves \\
\hline Anlodipino + lbuprofeno (5) & Ácido acetilsalicílico + Hidroclorotiazida (39) & Anlodipino + Sinvastatina (11) \\
Anlodipino + Diclofenaco (3) & Enalapril + Hidroclorotiazida (20) & Digoxina + Hidroclorotiazida (4) \\
Metformina + Nifedipino(3) & Captopril + Hidroclorotiazida (17) & Enalapril + Losartana(3) \\
\hline
\end{tabular}

Não houve correlação estatística entre potenciais interaçôes medicamentosas de qualquer gravidade e idade, gênero, estado civil, grau de escolaridade, renda mensal, percepção do estado de saúde, número de consultas médicas nos últimos quatro meses, número de médicos consultados nos últimos quatro meses, número de medicamentos consumidos, convênio de saúde, gasto com medicamentos nos últimos quatro meses, hipertensão arterial sistêmica, diabetes, doença pulmonar, doença reumática, doença cardíaca, câncer e doença mental.

Após ajuste dos dados no modelo multivariado, a variável que permaneceu associada a potenciais interaçôes moderadas foi o número de médicos consultados nos últimos dois meses. Para interaçôes graves, encontrou-se o número de internaçôes nos últimos quatro meses como fator mais significativo (Tabela 1).

Tabela 1. Fatores associados a potenciais interações medicamentosas moderadas e graves encontrados em uma amostra representativa dos idosos cadastrados na ESF do município de Timóteo, MG.

\begin{tabular}{ccc}
\hline & PIM moderadas & PIM graves \\
\hline Internação hospitalar nos últimos 4 meses. & - & OR $=2,65$ \\
& & IC 95\% $=1,10$ a 9,70 \\
& Valor- $p=0,049$ \\
Número de médicos consultados nos últimos 2 meses. & IC $95 \%=1,03$ a 5,32 & - \\
& Valor- $\mathrm{p}=0,037$ & \\
\hline
\end{tabular}

\section{Discussão}

A prevalência de potenciais interações medicamentosas encontradas neste estudo $(55,6 \%)$ foi semelhante à descrita na literatura, tendo em vista que os valores podem ser bastante variáveis e revelam alta prevalência entre idosos. Nos Estados Unidos, $27 \%$ de uma população geriátrica ambulatorial utilizava medicamentos com potencial para uma ou mais interaçôes medicamentosas. ${ }^{19} \mathrm{Na}$ Europa, 1601 indivíduos de uma população idosa de seis países foram investigados, verificando-se o consumo médio de sete fármacos por pessoa e $46 \%$ de potenciais interaçôes medicamentosas. ${ }^{20}$ No México, encontraram-se $80 \%$ de prescriçôes com potencial para ocorrência de interaçôes medicamentosas entre indivíduos com 50 anos ou mais. ${ }^{13}$ Estudo brasileiro transversal de base populacional realizado em São Paulo demonstrou prevalência de 26,5\% de potenciais interaçôes medicamentosas, 50,7\% tinham idade maior ou igual a 75 anos, $71,7 \%$ relataram apresentar regular ou ruim estado de saúde e $65,8 \%$ utilizavam de 2 a 5 fármacos, sendo que a maioria das interaçóes era de moderada gravidade, como encontrado em nosso estudo.?

As classes terapêuticas mais frequentemente envolvidas neste trabalho foram anti-inflamatórios e, principalmente, fármacos utilizados em doenças cardiovasculares. Dados da literatura revelam que as classes farmacológicas envolvidas mais frequentemente são os fármacos que atuam nos sistemas cardiovascular, gastrointestinal, nervoso e musculoesquelético. ${ }^{14}$

As PIML mais frequentes ocorreram entre anlodipino e diclofenaco e entre anlodipino e ibuprofeno, que representam duas associaçôes de anti-hipertensivos com anti-inflamatórios. A associação desses medicamentos, quando necessária, deve ser feita pelo menor tempo e dose possíveis. Pelo potencial de causar hemorragia gastrointestinal e/ou antagonismo/diminuição do efeito hipotensivo, os médicos devem tomar as devidas precauçóes para evitá-la. 
A PIML entre metformina e nifedipino apresenta tendência de queda da prevalência, uma vez que o nifedipino foi excluído da Relação Nacional de Medicamentos Essenciais do Ministério da Saúde (2012) como agente anti-hipertensivo. ${ }^{21}$ Atualmente, existe a tendência em substituir o nifedipino pelo anlodipino, fármaco com melhor posologia e perfil de efeitos adversos. A associação entre metformina e nifedipino poderia causar aumento da absorção da primeira, aumentando o risco de seus efeitos indesejáveis.

A PIMM entre ácido acetilsalicílico e hidroclorotiazida é uma interação com repercussão clínica discutível, pois o programa para análise das interaçóes medicamentosas não determina a dose utilizada das medicaçóes; portanto, não podemos afirmar se a dose considerada do ácido acetilsalicílico é a antitrombótica ou anti-inflamatória. Apesar de poder causar redução da eficácia diurética e anti-hipertensiva da hidroclorotiazida, acredita-se que o benefício relacionado à prevenção de doença cardiovascular dessa associaçáo seja superior ao risco.

As PIMM entre captopril e hidroclorotiazida e entre enalapril e hidroclorotiazida não contraindicam o uso dessas medicaçôes. Observa-se que, na prática clínica, o benefício da associação também supera o risco de hipotensão postural por vasodilatação e depleção do volume intravascular. ${ }^{22}$

A PIMG entre anlodipino e sinvastatina pode levar ao risco aumentado dos efeitos adversos da sinvastatina, uma vez que o anlodipino aumenta os níveis séricos da estatina por meio de alteraçôes em sua farmacocinética. É recomendado o uso de, no máximo, $20 \mathrm{mg}$ de sinvastatina quando em associação com anlodipino. ${ }^{23}$

A PIMG entre digoxina e hidroclorotiazida gera risco aumentado de intoxicação digitálica devido à hipocalemia induzida pelo diurético. Pode levar a arritmias, alteraçôes neurológicas e gastrointestinais. Sendo a digoxina um digitálico com baixo índice terapêutico, isto é, com doses tóxicas próximas às doses terapêuticas, é recomendável que as prescriçôes contendo esse medicamento sejam sempre verificadas quanto à possibilidade de ocorrência de interaçóes medicamentosas. ${ }^{24}$

A PIMG entre losartana e enalapril é reforçada pela VI Diretriz Brasileira de Hipertensão Arterial, que considera o benefício da associação dessas drogas inferior ao risco de insuficiência renal, hipercalemia e hipotensão. ${ }^{22}$

Quanto aos fatores de risco associados positivamente com a ocorrência de potenciais interaçóes medicamentosas, o uso de 6 ou mais fármacos e a condição de portadores de hipertensão arterial, diabetes mellitus ou problemas cardíacos foram encontrados em um estudo brasileiro. ${ }^{7}$ Idade maior que 60 anos, presença de 3 ou mais doenças crônicas e prescrição de 5 ou mais fármacos também foram variáveis significativamente associadas ao fenômeno. ${ }^{13} \mathrm{O}$ presente estudo demonstrou que a presença de internação hospitalar prévia e de automedicação também podem estar implicadas em uma maior chance de ocorrência de interaçóes graves e moderadas, respectivamente.

Como limitações deste estudo, ressaltam-se: (1) a impossibilidade, pelo tipo de desenho adotado (transversal), de verificação das repercussóes clínicas das PIM nas condições de saúde dos idosos; (2) a presença de interaçôes frequentemente utilizadas na prática clínica, cujo benefício parece superar o prejuízo, superestimando as taxas de prevalência, e (3) a utilização de apenas um software para análise das PIM (sabe-se que, apesar da maioria dos estudos utilizarem apenas um software para verificação de PIM, o uso de dois softwares aumenta a sensibilidade para a detecção desse evento). ${ }^{25}$

Por outro lado, o estudo é relevante ao destacar a importância epidemiológica do grande número de prescriçôes contendo anti-inflamatórios associados a anti-hipertensivos. Outrora considerados fármacos seguros e com uma ampla variedade de indicaçôes, sabe-se hoje que os anti-inflamatórios apresentam toxicidade cardiovascular, gastrointestinal e renal significativas, devendo ser utilizados no menor período de tempo e nas menores doses eficazes possíveis em idosos. ${ }^{26}$ Além disso, os resultados encontrados alertam para a presença de fatores que se somam aos já reconhecidamente capazes de se associarem a uma maior frequência de PIM entre idosos, tais como a presença de automedicação e de internação recente.

\section{Conclusões}

O presente estudo revelou uma alta prevalência de potenciais interaçóes medicamentosas $(55,6 \%)$ entre idosos, ratificando os dados descritos na literatura e demonstrando a alta frequência desse fenômeno. Não foram encontrados fatores associados a potenciais interaçóes medicamentosas relacionados a questôes sociodemográficas, condiçóes de saúde do idoso e consumo de medicamentos. Internaçấo hospitalar nos últimos quatro meses associou-se positivamente à ocorrência de potenciais interaçóes graves, e a prescrição de medicamentos por profissionais médicos associou-se a uma menor frequência de interaçôes de moderada gravidade. 
As classes terapêuticas mais frequentemente envolvidas em potenciais interaçóes medicamentosas foram anti-inflamatórios e, principalmente, fármacos utilizados em doenças cardiovasculares, em especial bloqueadores dos canais de cálcio e diuréticos.

Interaçôes farmacológicas podem comprometer a segurança e a saúde do paciente, sendo necessário cuidado quanto à prescrição de múltiplos medicamentos simultaneamente para assim evitar associaçóes que comprometam a qualidade de vida dos idosos.

Os dados do presente estudo foram fornecidos aos gestores de saúde e aos prescritores que atendiam nas equipes da ESF do município estudado para que tivessem conhecimento das características da populaçáo e do atendimento prestado. Dessa forma, esses profissionais poderiam elaborar planos e traçar metas para a melhoria da assistência à saúde em relação à ocorrência de potenciais interações medicamentosas entre idosos.

\section{Referências}

1. Karnikowski MG, Naves JO, Silver LD. Access to essential drugs in 11 Brazilian cities: a community-based evaluation and action method. Journal of Public Health Policy. 2004;25(3/4):288-98.

2. World Health Organization. Envelhecimento ativo: uma política de saúde. Gontijo S, translator. Brasília: Organização Pan-americana da Saúde; 2005. 60p.

3. Instituto Brasileiro de Geografia e Estatística (IBGE). Projeção da população. [acesso em 2013 Apr 06] Disponível em: http://www.ibge.gov.br.

4. Anderson GM, Beers MH, Kerluke K. Auditing prescription practice using explicit criteria and computerized drug benefit claims data. J Eval Clin Pract. 1997;3(4):283-94.

5. Flores VB, Benvegnú LA. Perfil de utilização de medicamentos em idosos da zona urbana de Santa Rosa, Rio Grande do Sul, Brasil. Cad. Saúde Pública. 2008;24(6):1439-1446. http://dx.doi.org/10.1590/S0102-311X2008000600024.

6. Katzung BG. Aspectos especiais da farmacologia geriátrica. In: Katzung BG, editor. Farmacologia básica \& clínica. $8^{a}$ ed. Rio de Janeiro: Guanabara-Koogan; 2002. p. 899-906.

7. Secoli SR, Figueiras A, Lebrão ML, Lima FD, Santos JL. Risk of potential drug-drug interactions among Brazilian elderly: a population-based, cross-sectional study. Drugs Aging. 2010;27(9):759-70.

8. Stockley $\mathrm{H}$. Interacciones Farmacológicas: fuente bibliográfica sobre interacciones, sus mecanismos, importancia clínica y orientación terapéutica. Barcelona: Pharma Editores; 2004. 831p.

9. Lazarou J, Pomeranz BH, Corey PN. Incidence of adverce drug reactions in hospitalized patients: a meta-analysis of prospective studies. JAMA. 1998;279(15):1200-1205

10. Einarson, TR. Drug-related hospital admissions. Ann Pharmacother. 1993;27:832-840.

11. Jha AK, Kuperman GJ, Rittenberg E, Teich JM, Bates DW. Identifying hospital admissions due to adverse drug events using a computer-based monitor. Pharmacoepidemiol Drug Saf. 2001;10:113-119.

12. Fonseca AL. Interações Medicamentosas. $3^{a}$ ed. Rio de janeiro: Editora e Publicações Biomédicas; 2001.

13. Doubova Dubova SV, Reyes-Morales H, Torres-Arreola LP, Suárez-Ortega M. Potential drug-drug and drug-disease interactions in prescriptions for ambulatory patients over 50 years of age in family medicine clinics in Mexico city. BMC Health Serv Res. 2007;7:147. http://dx.doi. org/10.1186/1472-6963-7-147

14. Bleich GW, Bleich A, Chiamulera P, Sanches ACC, Schneider DSLG, Teixeira JJV. Frequency of potential interactions between drugs in medical prescriptions in a city in southern Brazil. São Paulo Med J. 2009;127(4):206-210.

15. Daniel EF, Guarido CF. Ocorrência de possíveis interações medicamentosas em residências de um bairro do município de Marília, SP. Rev Bras Farm. 2009;90(1):54-58.

16. Loyola Filho Al, Uchoa E, Guerra HL, Firmo JOA, Lima-Costa MF. Prevalência e fatores associados à automedicação: resultados do Projeto Bambuí. Rev Saúde Pública. 2002;36(1):55-62.

17. Micromedex ${ }^{\circledast}$ Healthcare Series. Greenwood Village (CO): Thomson Reuters (Healthcare) Inc., 2007 [online]. Disponível em: https://www. thomsonhc.com/hcs/librarian/.

18. Drug Interactions Checker. Drug Information Online. Disponível em: http://www.drugs.com.

19. Costa AJ. Potential drug interactions in an ambulatory geriatric population. Fam Pract Barberton. 1991;8(3):234-236.

20. Bjokman IK, Schmidt IK, Bernsten CB. Drug-drug interactions in the elderly. Ann. Pharmacother. 2002;36(11):1675-1681.

21. Ministério da Saúde (BR), Secretaria de Ciência, Tecnologia e Insumos Estratégicos, Departamento de Assistência Farmacêutica e Insumos Estratégicos. Relação Nacional de Medicamentos Essenciais: RENAME. 8ª ed. Brasília: Ministério da Saúde; 2012.

22. VI Diretrizes Brasileiras de Hipertensão. Arq Bras Cardiol. 2010;95(Suppl1):I-III. http://dx.doi.org/10.1590/S0066-782X2010001700001. 
23. FDA Drug Safety Communication: new restrictions, contraindications, and dose limitations for Zocor (simvastatin) to reduce the risk of muscle injury. Disponível em: http://www.fda.gov/drugs/drugsafety/ucm256581.htm.

24. Osorio-de-Castro CGS. Interações medicamentosas. In: Fuchs FD, Wannmacher L. Farmacologia Clínica: fundamentos da terapêutica racional. $4^{a}$ ed. Rio de Janeiro: Guanabara Koogan; 2010. p. 115-123.

25. Vonbach P, Dubied A, Krähenbühl S, Beer JH. Evaluation of frequently used drug interaction screening programs. Pharm World Sci. 2008;30(4):367-74.

26. Pinheir RM, Wannmacher L. Uso racional de anti-inflamatórios não-esteróides. In: Ministério da Saúde (BR), Secretaria de Ciência, Tecnologia e Insumos Estratégicos. Uso racional de medicamentos: temas selecionados. Brasília: Ministério da Saúde; 2012. p. $41-50$. 